\title{
Treatment of duodenal peptic ulcer perforation by endoscopic clips: A novel approach
}

\author{
Mohan Achyut Joshi, Minakshi Gadhire ${ }^{1}$, Ashwini Arun Paranjpe ${ }^{1}$ \\ Department of Surgery, Gastroenterology Surgical and Endoscopy Services, Lokmanya Tilak Municipal Medical College and Hospital, \\ ${ }^{1}$ Department of Surgery, Lokmanya Tilak Municipal Medical College and Hospital, Mumbai, Maharashtra, India
}

Abstract
Aims and Objectives: In an attempt to avoid the high morbidity and mortality associated with
exploratory laparotomy in a patient of duodenal ulcer perforation (DUP), an attempt was made
to treat patients with spontaneous DUP with endoscopic clips. Background: DUP is a common
cause of surgical emergency. Traditional form of treatment includes nasogastric decompression,
fluid resuscitation, and exploratory laparotomy or laparoscopy for repair. Considering the
compromised state of such patients, endoscopic treatment and avoiding surgery will play a
great role in preventing the further stress caused by surgery and facilitate quicker recovery. On
thorough review of literature, we found few articles stating successful endoscopic clipping of
iatrogenic duodenal perforation occurred during upper endoscopy. Our case series appears to
be first of its kind reporting the use of endoclips for treating spontaneous (noniatrogenic) DUP.
Materials and Methods: Three young male patients presenting with a short duration history
of abdominal pain and vomiting and chest radiograph suggesting pneumoperitoneum were
selected for endoscopic clipping. All of them were hemodynamically stable with near normal
blood pressure and pulse rate. Under local anesthetic spray and mild sedation, endoscopic
clipping was carried out along with supportive treatment. Postoperatively, patients were
monitored clinically and radiographically and discharged after they could tolerate full diet.
Results and Conclusion: We recommend that in selected group of young patients of DUP who
have presented early and are hemodynamically stable, endoclips may be applied to approximate
mucosa. With supportive conservative treatment offered, thereafter healing of perforation
occurs without the need for laparotomy or laparoscopy for suturing the same.

\section{Introduction}

Duodenal ulcer perforation (DUP) is a common surgical emergency. ${ }^{[1]}$ The classic, pedicled omental patch that is performed for the "plugging" of these perforations was first described by Cellan-Jones in 1929. ${ }^{[2]}$ Although it is commonly attributed to Graham, who described the use of a free graft of

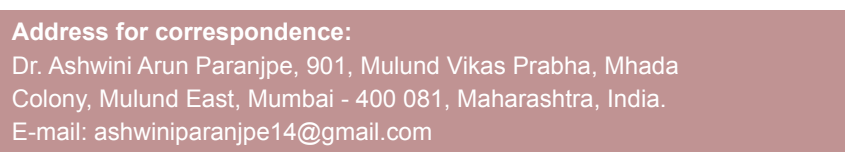

\begin{tabular}{|l|c|}
\hline \multicolumn{2}{|c|}{ Access this article online } \\
\hline \multirow{2}{*}{$\begin{array}{l}\text { Website: } \\
\text { www.jdeonline.in }\end{array}$} & Quick Response Code \\
\hline DOI: & \\
10.4103/jde.JDE_69_16 & \\
&
\end{tabular}

the omentum to repair the perforation in $1937 .{ }^{[3]}$ We report a case series of carefully selected patients treated successfully with endoscopic clipping which precludes laparotomy or laparoscopy which needs general anesthesia. It offers the advantages of a natural orifice approach.

\section{Materials and Methods}

Three young male patients with age ranging between 22 and 27 years were taken up for the treatment of DUP with endoclips.

This is an open access article distributed under the terms of the Creative Commons Attribution-NonCommercial-ShareAlike 3.0 License, which allows others to remix, tweak, and build upon the work non-commercially, as long as the author is credited and the new creations are licensed under the identical terms.

For reprints contact: reprints@medknow.com

How to cite this article: Joshi MA, Gadhire M, Paranjpe AA. Treatment of duodenal peptic ulcer perforation by endoscopic clips: A novel approach. J Dig Endosc 2017;8:24-6. 
All the three patients presented with acute upper abdominal pain with vomiting of 4-6 h duration. The diagnosis of peptic ulcer perforation was made by clinical history and chest $\mathrm{x}$-ray revealing free pneumoperitoneum [Figure 1]. All of them were hemodynamically stable with near normal blood pressure and pulse rate. All three underwent sonography of the abdomen which showed minimal free fluid in the Morrison's pouch. Complete hemogram, renal function tests, blood group, and cross matching, were done for all patients in anticipation of surgery under general anesthesia if clipping fails. All the three patients were explained the new concept of clipping for DUP by endoclips. The need for formal laparotomy if required either immediately or later was explained. Written informed consent was obtained. An abdominal drain was passed from the right side under local anesthesia before the patient underwent upper endoscopy to allow the air to come out from the greater sac as a result of inflation of air while endoscopy and clipping was done. The endoscopy was done under local anesthesia spray and sedation. The DUP was identified and perforation was confirmatively seen on the anterior wall of duodenum [Figure 2]. The perforation was clipped by two clips in two patients [Figures 3 and 4] and

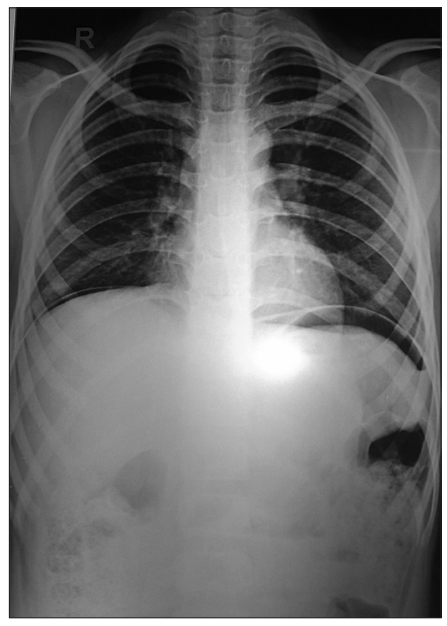

Figure 1: Chest radiograph suggestive of free gas under both domes of diaphragm

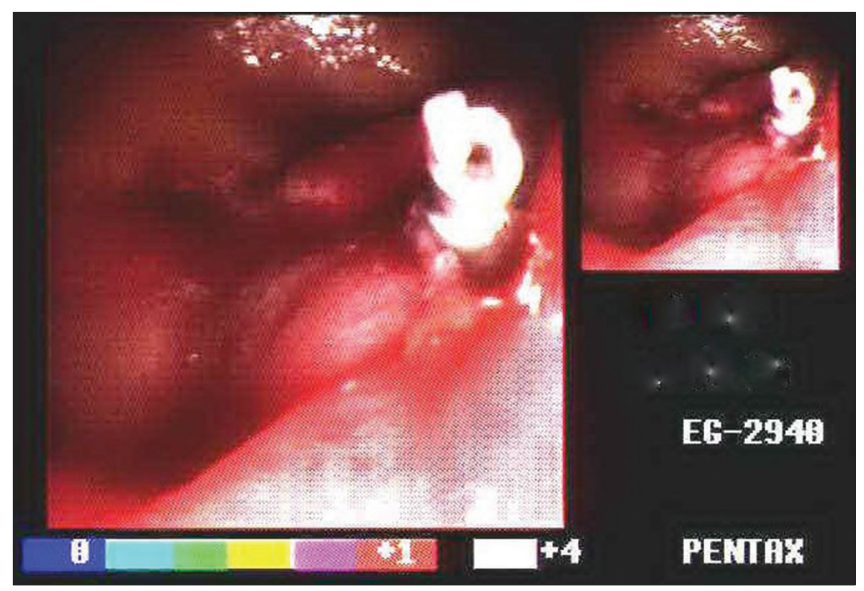

Figure 3: Endoscopic view of one clip applied on duodenal perforation three clips in one patient. Supportive treatment included nasogastric aspirations, IV fluids, proton pump inhibitors, and antibiotics. At the end of 5 days, methylene blue was given through the nasogastric tube. No bluish discoloration was seen in the drain in any patient. On the $6^{\text {th }}$ day, the patient was started on clear liquids orally. Bowel movements were ensured clinically and by auscultation. On the $7^{\text {th }}$ day, soft diet was started. The drain was removed subsequently. All patients showed uneventful recovery. At the end of 6 weeks, all underwent upper endoscopy which revealed healed ulcer with no clips.

\section{Discussion}

DUP is a common surgical emergency. ${ }^{[1]}$ It has good prognosis with timely and appropriate intervention. The gold standard for treatment of iatrogenic duodenal perforation is a surgical repair. ${ }^{[4-6]}$ A recent trend is toward the use of minimally invasive approach. In some cases, conservative medical management may be used for small perforations associated with unconfirmed or small leaks. ${ }^{[4-6]}$ The latest alternative to surgical or medical management of iatrogenic perforation is the endoscopic clipping device. On thorough review of literature,

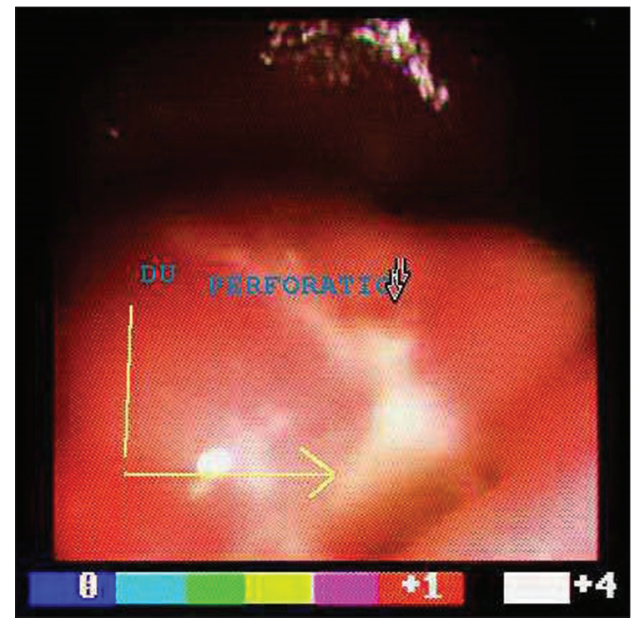

Figure 2: Endoscopic view of duodenal ulcer perforation

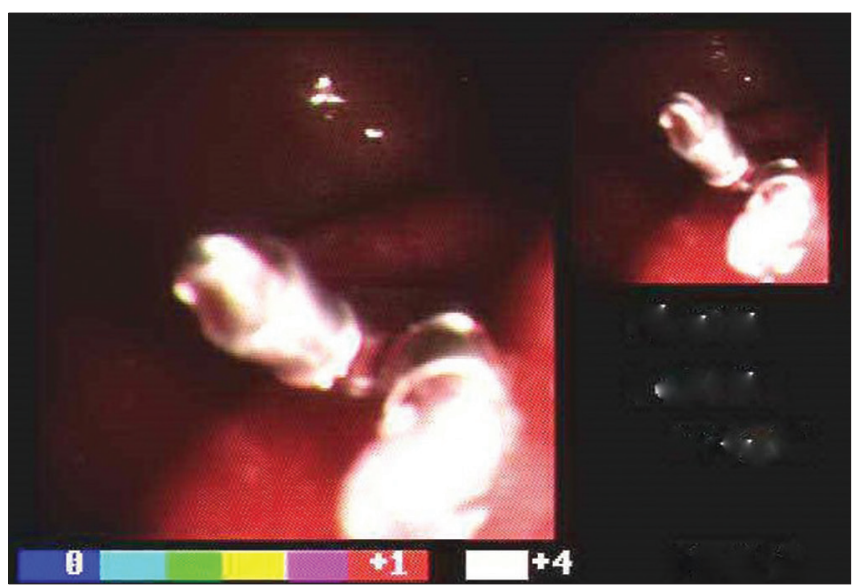

Figure 4: Endoscopic view of two clips applied on duodenal perforation 
we found few articles stating successful endoscopic clipping of duodenal perforations which were iatrogenic in nature. Our case series appears to be one of the few studies reporting endoscopic clipping of spontaneous DUP.

In 1993, endoclips were used for the first time to close an iatrogenic perforation secondary to resection of gastric leiomyoma. ${ }^{[7]}$ Kaneko and colleagues ${ }^{[8]}$ have used endoclips to treat duodenal perforation secondary to therapeutic endoscopy. Furthermore, cases reported by Sebastian et al. ${ }^{\left[{ }^{[9]}\right.}$ and others have demonstrated successful closure of an iatrogenic duodenal perforation with an endoscopic clipping device following EUS examination. ${ }^{[8,9]}$ No complications were reported in these case reports. Complete healing of the perforation site was observed by a 1-month follow-up endoscopy, and the clips were observed to dislodge spontaneously within 3 weeks of placement. ${ }^{[10]}$

A similar study by Goenka et al.$^{[1]}$ reports the successful use of over-the-scope clips for the closure of GI leaks and fistulas in six patients out of which three were inflammatory in origin. The site of lesion varied including lower esophagus, gastric body, and splenic flexure. The first sites healed completely only with the clips while closure of colonic perforation also required insertion of self-expandable metallic stent. Surgery was not required in any of the three patients.

Immediate identification and closure of the perforation is the key to achieving success with endoclips. ${ }^{[12]}$ Instant closure of the intestinal tear reduces, in theory, the spread of digestive enzymes, bile, and bacteria to peritoneum. With less inflammatory damage and bacterial contamination, antibiotics and symptomatic management may be sufficient to prevent further morbidity. ${ }^{[12]}$ In the above case reports, the intestinal perforation has been closed by endoclips using a through-the-scope system, which has certain limitations. Due to the low closure force and the limited opening distance between the clip jaws, multiple clips are required to close the defect. We used two clips in two and three in one case.

Recent studies recommend the use of an endoclipping device for the management of iatrogenic gastrointestinal perforations in select cases that fulfill the following criteria: instant identification of the perforation during the procedure; a tear that is $<10 \mathrm{~mm}$ in size; an endoscopy team that is experienced with using endoclips; and the availability of surgical help. ${ }^{[12]}$ After the procedure, the patient should be admitted to the hospital for medical management including nil-per-os, nasogastric suctioning, and intravenous fluids and antibiotics. ${ }^{[12]}$ Moreover, the patient should be closely monitored for signs of peritonitis, subcutaneous emphysema, hemodynamic instability, and sepsis. ${ }^{[12]}$ We followed the same protocol more stringently. Imaging studies should be performed $24 \mathrm{~h}$ after the procedure, and a liquid diet may be started if the imaging study does not show any leakage of contrast. ${ }^{[12,13]} \mathrm{In}$ our series, methylene blue was given through the nasogastric tube, and the drain was inspected for blue discoloration to identify the leak from DUP.

Although there were no complications associated with the use of clips for the closure of perforated duodenal ulcers, more studies are required to confirm the indications and safety associated with them.

We recommend that in a certain select group of young patients of DUP who have presented early and are hemodynamically stable, endoscopic clips may be applied to approximate mucosa. With supportive conservative treatment offered, thereafter healing of perforation occurs without the need for laparotomy or laparoscopy for suturing the same.

\section{Financial support and sponsorship}

Nil.

\section{Conflicts of interest}

There are no conflicts of interest.

\section{References}

1. Gupta S, Kaushik R, Sharma R, Attri A. The management of large perforations of duodenal ulcers. BMC Surg 2005;5:15.

2. Cellan-Jones CJ. A rapid method of treatment in perforated duodenal ulcer. Br Med J 1929;1:1076-7.

3. Graham RR. The treatment of perforated duodenal ulcers. Surg Gynecol Obstet 1937;64:235-8.

4. Chaudhary A, Aranya RC. Surgery in perforation after endoscopic sphincterotomy: Sooner, later or not at all? Ann R Coll Surg Engl 1996;78:206-8.

5. Bell RC, Van Stiegmann G, Goff J, Reveille M, Norton L, Pearlman NW. Decision for surgical management of perforation following endoscopic sphincterotomy. Am Surg 1991;57:237-40.

6. Charabaty-Pishvaian A, Al-Kawas F. Endoscopic treatment of duodenal perforation using a clipping device: Case report and review of the literature. South Med J 2004;97:190-3.

7. Binmoeller KF, Grimm H, Soehendra N. Endoscopic closure of a perforation using metallic clips after snare excision of a gastric leiomyoma. Gastrointest Endosc 1993;39:172-4.

8. Kaneko T, Akamatsu T, Shimodaira K, Ueno T, Gotoh A, Mukawa K, et al. Nonsurgical treatment of duodenal perforation by endoscopic repair using a clipping device. Gastrointest Endosc 1999;50:410-3.

9. Sebastian S, Byrne AT, Torreggiani WC, Buckley M. Endoscopic closure of iatrogenic duodenal perforation during endoscopic ultrasound. Endoscopy 2004;36:245.

10. Haider S, Kahaleh M. The use of endoscopic clipping devices in the treatment of iatrogenic duodenal perforation. Gastroenterol Hepatol (NY) 2010;6:660-1.

11. Goenka MK, Rai VK, Goenka U, Tiwary IK. Endoscopic management of gastrointestinal leaks and bleeding with the over-the-scope clip: A prospective study. Clin Endosc 2017;50:58-63.

12. Khokhar OS, Nguyen DT, Johnson L, Jha R, Haddad NG. Endoscopic management of iatrogenic duodenal perforation with linear-probe echoendoscope. Gastroenterol Hepatol (NY) 2010;6:657-9.

13. Mangiavillano B, Viaggi P, Masci E. Endoscopic closure of acute iatrogenic perforations during diagnostic and therapeutic endoscopy in the gastrointestinal tract using metallic clips: A literature review. J Dig Dis 2010;11:12-8. 